\title{
UNIFORM ERROR ESTIMATES OF OPERATIONAL QUADRATURE METHODS FOR NONLINEAR CONVOLUTION EQUATIONS ON THE HALF-LINE
}

\author{
P. P. B. EGGERMONT AND CH. LUBICH
}

\begin{abstract}
We study uniform error estimates of operational quadrature methods for nonlinear convolution equations on the half-line. Equations of this kind arise in control engineering and diffusion problems. The essential ingredients are the stability of the operational quadrature method in an $L^{2}$ setting, which is inherited from the continuous equation by its very construction, and a theorem that says that the behavior of the linearized equations is the same in all $L^{p}$ spaces $(1 \leq p \leq \infty)$.
\end{abstract}

\section{INTRODUCTION}

1.1. Problem statement. In this article we study quadrature methods for the numerical solution of nonlinear Volterra convolution equations

$$
x(t)+\int_{0}^{t} k(t-\tau) g(\tau, x(\tau)) d \tau=f(t), \quad t \in(0, \infty) .
$$

We derive uniform error estimates over the whole half-line in situations where problem (1.1) behaves stably. As a criterion for bounded input-bounded output stability of $(1.1)$ we find that the classical $L^{2}$ Circle Condition Theorem extends to $L^{\infty}$ (in fact, to all $L^{p}$ spaces with $1 \leq p \leq \infty$ ). Precise assumptions on $k$ and $g$ will be formulated later, but here we remark that weakly singular kernels like $k(t)=t^{-1 / 2}$ and smooth kernels as, e.g., $e^{-t}$ or cos $t$ are equally admitted throughout the paper. For convenience we limit our attention to scalar equations (1.1), although this restriction is not really essential.

Problems of the type considered here arise as feedback systems in control engineering, as boundary integral equations in diffusion problems, and in viscoelasticity. See Desoer and Vidyasagar [6], Corduneanu [4], Cannon [3], Ghez [8], Pipkin [16], Lodge et al. [12], Linz [11], Brunner and van der Houwen [2], and references therein.

We remark that for the special case $k(t)=1$ our results give estimates of linear multistep methods applied to nonlinear differential equations which may be arbitrarily stiff. This aspect will be further elaborated in a subsequent article.

Received May 5, 1989.

1980 Mathematics Subject Classification (1985 Revision). Primary 65R20, 45D05, 47A10. 
1.2. Operational quadrature methods. A discretization of (1.1) should conserve the convolution structure (which via fast Fourier transform techniques allows for computational efficiency) and should be able to follow the long-time behavior of the continuous problem (1.1), which to a large extent is determined by conditions on the range of the Laplace transform of the kernel,

$$
K(s)=\int_{0}^{\infty} e^{-s t} k(t) d t, \quad \operatorname{Re} s>0 .
$$

Here we study operational quadrature methods. They replace (1.1) on an equidistant grid $t_{n}=n h(n=0,1,2, \ldots)$ by a discrete recurr`nce relation

$$
x_{n}+h \sum_{j=0}^{n} k(n-j, h) g\left(t_{j}, x_{j}\right)=f_{n}, \quad n \geq 0,
$$

where $x_{n}$ is to approximate $x\left(t_{n}\right)$. Here the weights $k(n, h)$ are defined as the coefficients of the generating function (see Lubich [14])

$$
h \sum_{n=0}^{\infty} k(n, h) \zeta^{n}=K\left(\frac{\delta(\zeta)}{h}\right), \quad|\zeta|<1,
$$

where $\delta(\zeta)$ is the quotient of the generating polynomials of a linear multistep method. A practical choice is provided by backward differentiation formulas of orders $1 \leq m \leq 6$, which correspond to

$$
\delta(\zeta)=\sum_{l=1}^{m} \frac{(1-\zeta)^{l}}{l}
$$

The right-hand side $f_{n}$ in (1.2) equals essentially $f\left(t_{n}\right)$, but may also contain a correction of the convolution quadrature by a linear combination of $h g\left(t_{j}, x_{j}\right)$ for a few of the first $j=0,1, \ldots, J$. This possibility is included in order to take account of the behavior of $g(t, x(t))$ near $t=0$, similar to the familiar endpoint corrections of the trapezoidal rule.

Under reasonable assumptions it is known that $x_{n}$ approximates $x\left(t_{n}\right)$ with the same order as the underlying multistep method $\left(O\left(h^{m}\right)\right.$ for (1.4)), uniformly on bounded intervals. We also remark that $k(n, h)$ approximates $k(n h)$ for $n h$ bounded away from 0 with the order of the multistep method, again under suitable assumptions on $K(s)$.

Of course, to apply method (1.2-3), the Laplace transform $K(s)$ of the kernel needs to be known. In many applications this is no restriction: In diffusion problems the analytical derivation of the integral equation first yields $K(s)$, from which $k(t)$ is only then obtained by Laplace inversion (if at all). Also, modelling of feedback systems is done in terms of the transfer function $K(s)$ rather than the kernel $k(t)$. We emphasize that explicit knowledge of $k(t)$ is not necessary .

A FORTRAN program for equation (1.1) with $k(t)=t^{-1 / 2}$, based on BDF methods (1.4), is given in Hairer et al. [9]. That paper describes also a fast solution technique for the discrete nonlinear convolution equation (1.2) which requires $O\left(N(\log N)^{2}\right)$ operations to compute $x_{0}, \ldots, x_{N}$, based on fast Fourier 
transforms. The computation of the quadrature weights (1.3) for general kernels again uses fast Fourier transforms and is described in Lubich [14]. An implementation of equation (1.2) as a finite-term recurrence relation is possible if the generating function $(1.3)$ is rational.

1.3. Outline of the paper. Our aim in this article is to derive uniform error estimates for the discretization (1.2) of equation (1.1) over intervals of arbitrary length. As might be expected, this involves problems of consistency and $l^{\infty}$ stability. The problem of quadrature errors over the half-line is well understood with the techniques (and under the assumptions) of Lubich [14]. It is really the $l^{\infty}$ stability problem on which we have to concentrate here.

For equations of the above type (1.1), there is a well-developed theory in an $L^{2}$ setting. The Circle Condition Theorem (Theorem 2.1 below) says that if there exists a circle in the complex plane such that $\frac{\partial g}{\partial x}$ takes all its values inside the circle and $-1 / K(s)$ takes all its values outside of it for $\operatorname{Re} s \geq 0$, then we have an $L^{2}$ estimate

$$
\|x-\tilde{x}\|_{L^{2}\left(\mathbb{R}^{+}\right)} \leq c\|f-\tilde{f}\|_{L^{2}\left(\mathbb{R}^{+}\right)}
$$

for solutions $x, \tilde{x}$ corresponding to right-hand sides $f, \tilde{f}$ in (1.1). It turns out (Theorem 2.3) that under exactly the same conditions on $K$ and $g$ as in the continuous case, solutions of the discrete equation (1.2) satisfy the $l^{2}$ analogue of (1.5) with the same constant $c$ as above for all $h>0$ if the underlying multistep formula is $A$-stable, i.e., if

$$
\operatorname{Re} \delta(\zeta) \geq 0 \text { for }|\zeta|<1 .
$$

We obtain $l^{2}$ stability estimates for $A(\pi-\theta)$-stable methods with $\theta>\frac{\pi}{2}$, i.e., those satisfying

$$
|\arg \delta(\zeta)| \leq \theta \text { for }|\zeta|<1,
$$

if $K(s)$ satisfies the circle condition in the sector $|\arg s| \leq \theta$ instead of just $\operatorname{Re} s \geq 0$. The tools in $\S 2$ are Parseval's formula and the Banach contraction principle.

The key step in the transition from $L^{2}$ to $L^{\infty}$ in (1.5) is made in $\S 3$. Theorem 3.1 in its simplest form states that if the equation

$$
y(t)+\int_{0}^{t} b(t-\tau) a(\tau) y(\tau) d \tau=f(t), \quad t>0,
$$

is (uniquely) solvable in $L^{2}\left(\mathbb{R}^{+}\right)$for every $f \in L^{2}\left(\mathbb{R}^{+}\right)$, then it is also (uniquely) solvable in $L^{\infty}\left(\mathbb{R}^{+}\right)$for every $f \in L^{\infty}\left(\mathbb{R}^{+}\right)$. Here $b \in L^{1}\left(\mathbb{R}^{+}\right)$and $a \in$ $L^{\infty}\left(\mathbb{R}^{+}\right)$. Moreover, there is an important uniformity: If we let $a$ vary in some bounded subset $\mathscr{A}$ of $L^{\infty}\left(\mathbb{R}^{+}\right)$, and if the solutions of (1.6) satisfy

$$
\|y\|_{L^{2}\left(\mathbb{R}^{+}\right)} \leq c\|f\|_{L^{2}\left(\mathbb{R}^{+}\right)},
$$

with a constant $c$ which is independent of $a \in \mathscr{A}$, then also

$$
\|y\|_{L^{\infty}\left(\mathbb{R}^{+}\right)} \leq c^{\prime}\|f\|_{L^{\infty}\left(\mathbb{R}^{+}\right)},
$$


for some constant $c^{\prime}<\infty$ which can be chosen independently of $a \in \mathscr{A}$. This will allow us to obtain $L^{\infty}$ estimates for linearizations of (1.1) around arbitrary functions $x$. There is a similar uniformity with respect to certain classes of $L^{1}$ kernels $b$. For our purposes this will be particularly important in the discrete analogue (Theorem 4.1), because it leads to $l^{\infty}$ estimates. Theorems 3.1 and 4.1 generalize results of Eggermont [7], where an extra assumption $b(t)=O\left(t^{-\alpha-1}\right)$ as $t \rightarrow \infty$ was needed. Here "just" $b \in L^{1}\left(\mathbb{R}^{+}\right)$is enough.

Section 5 contains the $L^{\infty}$ version of the Circle Condition Theorem: Solutions of (1.1) satisfy an estimate $(1.5)$ with $L^{2}$ replaced by $L^{\infty}$,

$$
\|x-\tilde{x}\|_{L^{\infty}\left(\mathbb{R}^{+}\right)} \leq c^{\prime}\|f-\tilde{f}\|_{L^{\infty}\left(\mathbb{R}^{+}\right)},
$$

if the assumptions of the $L^{2}$ Circle Condition Theorem are met and, as a necessary extra condition, if the linear equation $(1.1)$, with $g(t, x)=\lambda x$ and $\lambda$ the center of the circle, has an integrable resolvent. The ingredients of the proof are as follows: We first apply the $L^{2}$ Circle Condition Theorem to linearizations of (1.1) around arbitrary functions $x(t)$. With Theorem 3.1 we then obtain $L^{\infty}$ estimates for the solutions of the linearized equations, uniformly in $x$. The mean value theorem then allows us to pass from the linearizations to the nonlinear problem.

In $\S 6$ we derive $l^{\infty}$ stability estimates for solutions of the discretized equation (1.2). This uses the results and techniques of the preceding sections. To verify the conditions of Theorem 4.1, our analysis requires some assumptions on $K(s)$ in addition to those of the $L^{\infty}$ Circle Condition Theorem 5.1. They do, however, not seem restrictive for the above-mentioned applications.

In $\S 7$ we combine the $l^{\infty}$ stability estimates with results on the quadrature error to obtain at last uniform convergence over the whole half-line, of the order of the underlying multistep method.

Finally, in $\S 8$ we give the Circle Condition Theorem in $L^{p}$ for $1 \leq p \leq \infty$. The main ingredients here are the $M$. Riesz convexity theorem, and once more Theorem 3.1.

\section{The $L^{2}$ Circle Condition Theorem and $l^{2}$ Stability OF THE DISCRETIZATION}

We consider equation (1.1) denoted as

$$
x+k * g(x)=f \text { in } L^{2}\left(\mathbb{R}^{+}\right),
$$

where * denotes convolution, and $[g(x)](t)=g(t, x(t))$. The following conditions on $k$ and $g$ will be assumed throughout:

$k$ is locally integrable and has a Laplace transform $K$ which is

a continuous function from $\overline{\mathbb{C}}^{+}$into $\overline{\mathbb{C}}$.

Here $\overline{\mathbb{C}}=\mathbb{C} \cup\{\infty\}$ is the Riemann sphere, and $\overline{\mathbb{C}}^{+}$denotes the closed right half-plane including infinity.

$g$ is continuously differentiable on $\mathbb{R}^{+} \times \mathbb{R}$, and $g(t, 0)=0$

for all $t \geq 0$. 
The last condition is no real restriction, since otherwise in (2.1) we replace $g(x)$ by $g(x)-g(0)$ and $f$ by $f-k * g(0)$. It simplifies, however, the statement of results. Existence of solutions $x \in L^{2}\left(\mathbb{R}^{+}\right)$will be shown for every $f \in L^{2}\left(\mathbb{R}^{+}\right)$, instead of $f-k * g(0) \in L^{2}\left(\mathbb{R}^{+}\right)$.

The following theorem is a classic in the control engineering literature, see Corduneanu [4], or Desoer and Vidyasagar [6]. Early versions of it are due to Sandberg [17] and Zames [19].

Theorem 2.1 (Circle Condition Theorem in $L^{2}$ ). In addition to the above assumptions on $k$ and $g$, suppose that for some real $\lambda, l$,

$$
\left|\frac{\partial g}{\partial x}(t, x)-\lambda\right| \leq l \text { for all } t, x
$$

and there exists a positive number $d$ such that

$$
\left|-\frac{1}{K(s)}-\lambda\right| \geq l+d \text { for all } \operatorname{Re} s \geq 0 .
$$

Then the integral equation (1.1) has a unique solution $x \in L^{2}\left(\mathbb{R}^{+}\right)$for every $f \in L^{2}\left(\mathbb{R}^{+}\right)$. Moreover, if $x$ and $\tilde{x}$ are the solutions of (1.1) corresponding to $f, \tilde{f} \in L^{2}\left(\mathbb{R}^{+}\right)$, then

$$
\|x-\tilde{x}\|_{L^{2}\left(\mathbb{R}^{+}\right)} \leq c\|f-\tilde{f}\|_{L^{2}\left(\mathbb{R}^{+}\right)},
$$

with $c=(|\lambda|+l+d) / d$.

Remark. In the engineering literature the condition (2.3) is usually formulated in terms of the Nyquist diagram $\{K(i \omega): \omega \in \mathbb{R}\}$. In this case, $(2.3)$ is required to hold only for $\operatorname{Re} s=0$, and the condition for $\operatorname{Re} s>0$ comes from the additionally needed information that the Nyquist diagram does not encircle the negative reciprocal of the disk of (2.3), i.e., $\{-1 / z:|z-\lambda| \leq l\}$.

As a first major step in the proof we want to get rid of the (possibly) unbounded operator, embodied by the convolution with $k$, on $L^{2}\left(\mathbb{R}^{+}\right)$. Note that for $\lambda=0$, we have $|K(s)| \leq(l+d)^{-1}$ for all $\operatorname{Re} s \geq 0$, so assume $\lambda \neq 0$. We then define the resolvent $r_{\lambda}$ as the solution of

$$
r_{\lambda}+\lambda k * r_{\lambda}=\lambda k \text { on } \mathbb{R}^{+} \text {. }
$$

One verifies that $r_{\lambda}$ is locally integrable, e.g., by Picard iteration on arbitrary intervals $[0, T]$ for finite $T$, and that it has a Laplace transform $R_{\lambda}$ given by

$$
R_{\lambda}(s)=\lambda K(s) /[1+\lambda K(s)], \quad \operatorname{Re} s \geq 0 .
$$

It follows from the circle criterion that

$$
\left|R_{\lambda}(s)\right| \leq|\lambda| /(l+d), \quad \operatorname{Re} s \geq 0,
$$

and consequently that for every $x \in L^{2}\left(\mathbb{R}^{+}\right)$,

$$
\left\|r_{\lambda} * x\right\|_{L^{2}\left(\mathbb{R}^{+}\right)} \leq|\lambda| /(l+d) \cdot\|x\|_{L^{2}\left(\mathbb{R}^{+}\right)},
$$


by Young's inequality and Plancherel's formula, see Stein and Weiss [18, p. 178 and p. 17]. In other words, convolution with $r_{\lambda}$ is a bounded linear operation on $L^{2}\left(\mathbb{R}^{+}\right)$. It now follows that if $x$ satisfies equation (1.1), then it also satisfies

$$
x+\lambda^{-1} r_{\lambda} *(g(x)-\lambda x)=f-r_{\lambda} * f \text {, }
$$

and vice versa.

Proof of Theorem 2.1. We consider equation (2.7) and let

$$
\Phi(x)=\lambda^{-1} r_{\lambda} *(g(x)-\lambda x) .
$$

Then for $x, \tilde{x} \in L^{2}\left(\mathbb{R}^{+}\right)$we get by (2.6), the mean value theorem and condition (2.2) that

$$
\|\Phi(x)-\Phi(\tilde{x})\|_{L^{2}\left(\mathbb{R}^{+}\right)} \leq \frac{l}{l+d}\|x-\tilde{x}\|_{L^{2}\left(\mathbb{R}^{+}\right)},
$$

so that $\Phi$ is a strong contraction. It follows from the Banach contraction principle that (2.7), written as $x+\Phi(x)=v$, has a unique solution $x \in L^{2}\left(\mathbb{R}^{+}\right)$. Moreover, if $x$ and $\tilde{x}$ correspond to $v$ and $\tilde{v}$, then

$$
\|x-\tilde{x}\|_{L^{2}\left(\mathbb{R}^{+}\right)} \leq \frac{l+d}{d}\|v-\tilde{v}\|_{L^{2}\left(\mathbb{R}^{+}\right)} .
$$

Finally, if $v=f-r_{\lambda} * f$ and $\tilde{v}=\tilde{f}-r_{\lambda} * \tilde{f}$, then

$$
\|v-\tilde{v}\|_{L^{2}\left(\mathbb{R}^{+}\right)} \leq C\|f-\tilde{f}\|_{L^{2}\left(\mathbb{R}^{+}\right)},
$$

with $C=\sup _{\operatorname{Re} s \geq 0}\left|1-R_{\lambda}(s)\right| \leq(l+d+|\lambda|) /(l+d)$ by Plancherel's formula and (2.3). Putting it all together, we finally obtain (2.4).

We note a special case of the circle condition theorem where $\lambda$ can become arbitrarily large.

Corollary 2.2. Suppose that for some $\lambda>0, \rho<1$,

$$
\begin{aligned}
& \left|\frac{\partial g}{\partial x}-\lambda\right| \leq \rho \lambda \quad \text { everywhere, } \\
& \operatorname{Re} K(s) \geq 0 \text { for all } \operatorname{Re} s \geq 0 .
\end{aligned}
$$

Then equation (1.1) has a unique solution $x \in L^{2}\left(\mathbb{R}^{+}\right)$for every $f \in L^{2}\left(\mathbb{R}^{+}\right)$. Moreover, the estimate (2.4) holds with $c=(1-\rho)^{-1}$.

Remark. Corollary 2.2 is actually well known in the theory of general Hammerstein (integral) equations

$$
x+\mathscr{K} g(x)=f \quad \text { in } L^{2}
$$

with $g$ as in the corollary, and where $\mathscr{K}$ is densely defined and mootone, i.e.,

$$
\operatorname{Re}(x, \mathscr{K} x) \geq 0
$$

for all $x$ in the domain of $\mathscr{K}$. See Kolodner [10, §3.2]. Condition (2.12) is equivalent to $(2.10)$ when $\mathscr{K}$ is a convolution operator. When $(2.12)$ holds, the 
operator $\lambda(I+\lambda \mathscr{K})^{-1} \mathscr{K}$ has a bounded extension to all of $L^{2}$ and with norm bounded by 1 , for all $\lambda \geq 0$. This operator is the analogue of the resolvent $r_{\lambda}$ in (2.5). With this modification, the work of Kolodner is readily applied to (2.11).

We now turn to the discretized problem (1.2), keeping the previous assumptions on $k$ and $g$. We suppose that the discretization method (1.3) is $A$-stable, i.e.,

$$
\operatorname{Re} \delta(\zeta) \geq 0 \text { for }|\zeta|<1 .
$$

By Dahlquist's [5] order barrier, the order of the method, given as the number $m$ in the relation $h^{-1} \delta\left(e^{-h}\right)=1+O\left(h^{m}\right)$, cannot exceed 2 under condition (2.13). In many cases, this restriction is not really necessary. If $K(s)$ satisfies the circle condition (2.3) in a sector $|\arg s| \leq \theta$ with $\theta>\frac{\pi}{2}$, then the following result remains valid for $A(\pi-\theta)$-stable methods, i.e., for those with

$$
|\arg \delta(\zeta)| \leq \theta \text { for }|\zeta|<1 .
$$

We remark that the BDF methods (1.4) satisfy (2.13) for $m=1$ and 2 , and (2.14) with $\pi-\theta=88^{\circ}, 73^{\circ}, 51^{\circ}, 18^{\circ}$ for $m=3,4,5,6$, respectively.

Theorem 2.3. Under the same conditions on $k$ and $g$ as in the Circle Condition Theorem 2.1 and under the A-stability condition (2.13), the equation (1.2) has a unique solution $x=\left\{x_{n}\right\}_{n \geq 0} \in l^{2}$ for every $f=\left\{f_{n}\right\}_{n \geq 0} \in l^{2}$, for arbitrary $h>0$. Moreover, if $x, \tilde{x}$ correspond to $f, \tilde{f} \in l^{2}$, then

$$
\|x-\tilde{x}\|_{l^{2}} \leq c\|f-\tilde{f}\|_{l^{2}},
$$

with $c$ as in Theorem 2.1. In particular, $c$ is independent of $h$.

Proof. We define the discrete resolvent by (cf. (2.5))

$$
r_{\lambda}(n, h)+h \sum_{j=0}^{n} \lambda k(n-j, h) r_{\lambda}(j, h)=\lambda k(n, h), \quad n \geq 0 .
$$

Forming the generating function and using (1.3), we find

$$
h \sum_{n=0}^{\infty} r_{\lambda}(n, h) \zeta^{n}=R_{\lambda}\left(\frac{\delta(\zeta)}{h}\right),
$$

where again $R_{\lambda}(s)=\lambda K(s) /(1+\lambda K(s))$ is the Laplace transform of the continuous resolvent. By the A-stability condition (2.13) we have

$$
\sup _{|\zeta| \leq 1}\left|R_{\lambda}\left(\frac{\delta(\zeta)}{h}\right)\right| \leq \sup _{\operatorname{Re} s \geq 0}\left|R_{\lambda}(s)\right| \text { for all } h>0 .
$$

By Parseval's formula the left-hand expression bounds (and in fact equals) the $l^{2}$ norm of the convolution operator

$$
\left\{f_{n}\right\}_{n} \mapsto\left\{h \sum_{j=0}^{n} r_{\lambda}(n-j, h) f_{j}\right\}_{n},
$$


which is therefore bounded by $|\lambda| /(l+d)$ as in (2.6). Now as in the continuous case (2.7), equation (1.2) is equivalent to

$$
x_{n}+\lambda^{-1} h \sum_{j=0}^{n} r_{\lambda}(n-j, h)\left(g\left(t_{j}, x_{j}\right)-\lambda x_{j}\right)=f_{n}-h \sum_{j=0}^{n} r_{\lambda}(n-j, h) f_{j} \text {. }
$$

The interpretation of (2.17) is now that to obtain (2.18) we could have applied the operational quadrature method directly to equation (2.7). The proof proceeds now as that of Theorem 2.1.

Remark. The results of this (and the subsequent) sections are easily extended to systems of equations (1.1). An $L^{2}$ estimate (2.4) is obtained if there exists a matrix $A$ and an invertible matrix $B$ such that for some suitable matrix norm $\|\cdot\|$,

$$
\begin{gathered}
\left\|B^{-1}\left(\frac{\partial g}{\partial x}-A x\right)\right\| \leq l \text { everywhere, } \\
\left\|(I+K(s) A)^{-1} K(s) B\right\| \leq m \text { for } \operatorname{Re} s \geq 0,
\end{gathered}
$$

with $\operatorname{lm}<1$. The constant $c$ in $(2.4)$ is then $c=C /(1-\operatorname{lm})$ with $C=$ $\sup \left\|(I+K(s) A)^{-1}\right\|$, taken over $\operatorname{Re} s \geq 0$. The same constant still appears in the multidimensional version of Theorem 2.3.

\section{ON LINEAR CONVOLUTION-LIKE OPERATORS}

Let $b \in L^{1}(\mathbb{R})$, and let $e \in C(\mathbb{R})$ with $e(0)=0$. We consider the class $\mathscr{F}(b, e)$ of measurable functions $k$ on $\mathbb{R}^{2}$ for which there exists a positive constant $\mu$ such that

$$
|k(t, \tau)| \leq \mu b(\mu(t-\tau)), \quad \text { a.e. } t, \tau \in \mathbb{R},
$$

and

$$
\sup _{t} \int_{\mathbb{R}}|k(t+h, \tau)-k(t, \tau)| d \tau \leq e(\mu h) .
$$

Note that if $k(t, \tau)=b(t-\tau) a(\tau)$ for $a \in L^{\infty}(\mathbb{R}),\|a\|_{L^{\infty}(\mathbb{R})} \leq 1$, then $(3.1-2)$ are satisfied for a suitable function $e$.

Let $\Omega$ denote either $\mathbb{R}$ or $\mathbb{R}^{+}$(or any subset of $\mathbb{R}$, for that matter). Then $k$ generates a linear integral operator $\mathscr{K}$ defined by

$$
\mathscr{K} x(t)=\int_{\Omega} k(t, \tau) x(\tau) d \tau, \quad t \in \Omega,
$$

which maps any $L^{p}(\Omega)$ into itself for $1 \leq p \leq \infty$, and for all $x \in L^{p}(\Omega)$ we have

$$
\|\mathscr{K} x\|_{L^{p}(\Omega)} \leq\|b\|_{L^{\prime}(\mathbb{R})}\|x\|_{L^{p}(\Omega)} .
$$

This is essentially Young's inequality, Stein and Weiss [18, p. 179]. 
The class of operators $\mathscr{K}$ given by $(3.1-3)$ is also denoted by $\mathscr{F}(b, e)$. For such operators we may define the spectrum with respect to $L^{p}(\Omega)$ in the usual way by

(3.5) $\sigma^{p}(\mathscr{K})=\left\{\lambda \in \mathbb{C}: \lambda \mathscr{I}-\mathscr{K}\right.$ has no bounded inverse on $\left.L^{p}(\Omega)\right\}$.

We are interested in obtaining existence theorems for equations $\lambda x-\mathscr{K} x=f$ in $L^{\infty}(\Omega)$ starting from the $L^{2}(\Omega)$ theory, so we would like to show that $\sigma^{\infty}(\mathscr{K}) \subset \sigma^{2}(\mathscr{K})$, possibly even with equality. Since we are interested in nonlinear equations, we need some sort of uniformity in the above result. In Eggermont [7], the following theorem was proved for $p=2$ under the assumption

$$
b(t)=O\left(|t|^{-\alpha-1}\right), \quad|t| \rightarrow \infty ;
$$

here we show that we can do without it. The method of proof remains essentially the same, however.

Theorem 3.1. Let $\mathscr{E} \subset \mathscr{F}(b, e)$, let $\lambda \in \mathbb{C}$ and let $1<p<\infty$. If $\lambda \notin \sigma^{p}(\mathscr{K})$ for all $\mathscr{K} \in \mathscr{E}$ and

$$
\sup \left\|(\lambda \mathscr{I}-\mathscr{K})^{-1}\right\|_{L^{p}(\Omega)}<\infty,
$$

then $\lambda \notin \sigma^{\infty}(\mathscr{K})$ for all $\mathscr{K} \in \mathscr{E}$ and

$$
\sup \left\|(\lambda \mathscr{J}-\mathscr{K})^{-1}\right\|_{L^{\infty}(\Omega)}<\infty .
$$

Here the suprema are taken over all $\mathscr{K} \in \mathscr{E}$.

In this generality, the question what happens when $p=1$ is just beyond our reach. Even the simple case when $\mathscr{E}=\{\mathscr{K}\}$ is a singleton is not clear, but if the class $\mathscr{E}$ is essentially closed under adjugation, then the case $p=1$ can be handled as well; see $\S 8$. Perhaps this is the right time to mention the work of Barnes [1]. He considers the class of integral operators $\mathscr{K},(3.3)$, which (only) have the property that for some $\alpha>0$,

$$
\underset{t \in \Omega}{\operatorname{ess} \sup } \int_{|t-\tau| \geq n}(1+|t-\tau|)^{\alpha}\{|k(t, \tau)|+|k(\tau, t)|\} d \tau
$$

is bounded for $n=0$, and tends to zero as $n \rightarrow \infty$. For such operators Barnes proves [1, Theorem 4.8(2)] that $\sigma^{p}(\mathscr{K}) \cup \sigma^{p}\left(\mathscr{K}^{T}\right)$ is independent of $p, 1 \leq p \leq \infty$. Here, $\mathscr{K}^{T}$ is the integral operator with kernel $k(\tau, t)$ (as opposed to $k(t, \tau)$ for the operator $\mathscr{K})$. In particular, then, $\sigma^{p}(\mathscr{K}) \subset \sigma^{2}(\mathscr{K})$, $1 \leq p \leq \infty$. Compared to our work, the big difference is the absence of (an analogue of) condition (3.2).

We proceed to prove Theorem 3.1. We begin by breaking the proposition in two.

Proposition 3.2. Let $1<p<\infty$, let $\mathscr{E} \subset \mathscr{F}(b, e)$, and let $\lambda \in \mathbb{C}$. If there exists a constant $c>0$ such that for all $\mathscr{K} \in \mathscr{E}$ and for all $x \in L^{p}(\Omega)$,

$$
\|\lambda x-\mathscr{K} x\|_{L^{p}(\Omega)} \geq c\|x\|_{L^{p}(\Omega)},
$$


then likewise for some $c^{\prime}>0$, and for all $\mathscr{K} \in \mathscr{E}$ and for all $x \in L^{\infty}(\Omega)$,

$$
\|\lambda x-\mathscr{K} x\|_{L^{\infty}(\Omega)} \geq c^{\prime}\|x\|_{L^{\infty}(\Omega)} .
$$

Proposition 3.3. Let $1<p<\infty$; then for $\mathscr{K} \in \mathscr{F}(b, e)$,

$$
\sigma^{\infty}(\mathscr{K}) \subset \sigma^{p}(\mathscr{K}) .
$$

Using Propositions 3.2 and 3.3, we can now prove Theorem 3.1.

Proof of Theorem 3.1. Evidently, (3.7) implies (3.10), and thus also (3.11). From Proposition 3.3 we get that $\lambda \notin \sigma^{\infty}(\mathscr{K})$ for all $\mathscr{K} \in \mathscr{E}$ and thus $\lambda \mathcal{F}-\mathscr{K}$ is invertible on $L^{\infty}(\Omega)$. Combined with (3.11), this gives (3.8).

We now set out to prove Proposition 3.2. The proof of Proposition 3.3 will be omitted, since it is essentially the same as the proof of Lemma 4.3 in Eggermont [7]. The extra condition (3.6) is not used there.

We begin by proving some results about certain weight functions. They provide the crucial steps in the proof of Proposition 3.2.

Lemma 3.4. Let $\left\{t_{n}\right\}_{n} \subset \Omega$ be arbitrary, and set

$$
a_{n}(t)=\left(1+\left|\frac{t-t_{n}}{n}\right|^{2}\right)^{-1}, \quad t \in \mathbb{R} .
$$

Let $r=\left(2\|b\|_{L^{\prime}(\mathbb{R})}\right)^{-1}$, and let $w_{n}(t)$ be the solution of the convolution equation

$$
w_{n}(t)-r \int_{\mathbb{R}} b(\tau-t) w_{n}(\tau) d \tau=a_{n}(t), \quad t \in \mathbb{R},
$$

and $v_{n}(t)=\left[w_{n}(t)\right]^{1 / p}$, where $1<p<\infty$. Then the following holds, uniformly in $n$ and $t_{n}$ :

(i) $w_{n}(t)>0$ for all $t \in \mathbb{R}$;

(ii) $\left\|v_{n}\right\|_{L^{p}(\Omega)}=O\left(n^{1 / p}\right), n \rightarrow \infty$;

(iii) There exists $\delta>0$ such that $v_{n}(t)>\frac{1}{2}$ for all $t$ with $\left|t-t_{n}\right| \leq \delta$;

(iv) $\int_{\mathbb{R}} b(\tau-t) w_{n}(\tau) d \tau \leq r^{-1} w_{n}(t), t \in \mathbb{R}$;

(v) There exists a constant $c$ such that for all $t, \tau \in \mathbb{R}$ with $|t-\tau|<n$,

$$
\left|v_{n}(t)-v_{n}(\tau)\right| \leq c v_{n}(\tau)(|t-\tau| / n)^{1 / p} .
$$

Proof. Setting $\check{b}(t)=b(-t)$, we write $(3.13)$ as $w_{n}-r \check{b} * w_{n}=a_{n}$, with the solution given by $w_{n}=a_{n}+\beta * a_{n}$, where

$$
\beta=\sum_{k=1}^{\infty} r^{k} \check{b}^{* k} \in L^{1}(\mathbb{R}),
$$

in which $\check{b}^{* 1}=\check{b}$ and $\check{b}^{*(k+1)}=\check{b} * \check{b}^{* k}$. Since $\breve{b}(t) \geq 0$ for all $t$, then $w_{n}(t) \geq a_{n}(t)>0$ as well. This is (i). So $v_{n}$ is well defined, and $\left\|v_{n}\right\|_{L^{\prime}(\Omega)}^{p}=$ $\left\|w_{n}\right\|_{L^{\prime}(\Omega)}=2\left\|\dot{a}_{n}\right\|_{L^{\prime}(\Omega)} \leq 2 \pi n$. This is (ii). Part (iii) follows from $v_{n}(t) \geq$ $\left[a_{n}(t)\right]^{1 / p}$. Part (iv) follows from the equality $\check{b} * w_{n}=r^{-1}\left(w_{n}-a_{n}\right)$. To prove 
$(\mathrm{v})$, note that $a_{n}(t)$ satisfies

$$
\left|a_{n}(t)-a_{n}(\tau)\right| \leq c_{1} a_{n}(\tau)|t-\tau| / n
$$

for all $\tau, t \in \mathbb{R}$ with $|t-\tau| \leq n$. Then writing

$$
w_{n}(t)-w_{n}(\tau)=a_{n}(t)-a_{n}(\tau)+\int_{\mathbb{R}} \beta(r)\left[a_{n}(t-r)-a_{n}(\tau-r)\right] d r
$$

we obtain for $|t-\tau|<n$ that

$$
\begin{aligned}
\left|w_{n}(t)-w_{n}(\tau)\right| & \leq c_{1}|t-\tau| / n\left(a_{n}(\tau)+\int_{\mathbb{R}} \beta(r) a_{n}(\tau-r) d r\right) \\
& \leq c_{1} w_{n}(\tau)|t-\tau| / n .
\end{aligned}
$$

Finally, since $f(x)=x^{1 / p}(x>0)$ is Lipschitz continuous of order $\frac{1}{p}$, we get that for some constant $c_{2}$,

$$
\left|v_{n}(t)-v_{n}(\tau)\right|=\left|\left[w_{n}(t)\right]^{1 / p}-\left[w_{n}(\tau)\right]^{1 / p}\right| \leq c_{2}\left|w_{n}(t)-w_{n}(\tau)\right|^{1 / p}
$$

By the above estimate, (v) follows.

Lemma 3.5. Let $V(t)=\min \left\{|t|^{1 /(p-1)}, 1\right\}$ for $t \in \mathbb{R}$, and let

$$
l_{n}(t, \tau)=\frac{v_{n}(t)}{v_{n}(\tau)}-1
$$

Then

$$
\varepsilon_{n}=\int_{\mathbb{R}} b(t) V(t / n) d t \rightarrow 0 \text { as } n \rightarrow \infty,
$$

and

$$
B_{n}=\sup _{\tau \in \mathbb{R}} \int_{\mathbb{R}} b(t-\tau)\left|l_{n}(t, \tau)\right|^{p} /[V(|t-\tau| / n)]^{p-1} d t
$$

remains bounded as $n \rightarrow \infty$.

Proof. It is easy to see that $\varepsilon_{n} \rightarrow 0$ as $n \rightarrow \infty$ : since $V\left(\frac{t}{n}\right) \rightarrow 0$ as $n \rightarrow \infty$ for every $t$ (though not uniformly), the dominated convergence theorem does the trick. To estimate $B_{n}$, we need to split the integration interval. First of all, the part of the integral over $|t-\tau|<n$ is bounded by

$$
\int_{|t-\tau|<n} b(t-\tau) \frac{\left|l_{n}(t, \tau)\right|^{p}}{[|t-\tau| / n]} d t \leq c \int_{|t-\tau|<n} b(t-\tau) d t,
$$

by Lemma $3.4(\mathrm{v})$, and this is bounded by $c \cdot\|b\|_{L^{1}(\mathbb{R})}$. For the part of the integral over $|t-\tau|>n$ we first note that here $V(|t-\tau| / n)=1$, and secondly, since both $v_{n}(t)$ and $v_{n}(\tau)$ are always positive, that

$$
\left|l_{n}(t, \tau)\right|=\left|\frac{v_{n}(t)-v_{n}(\tau)}{v_{n}(\tau)}\right| \leq \frac{\max \left\{v_{n}(t), v_{n}(\tau)\right\}}{v_{n}(\tau)},
$$

and so surely

$$
\left|l_{n}(t, \tau)\right|^{p} \leq \frac{\left[v_{n}(t)\right]^{p}+\left[v_{n}(\tau)\right]^{p}}{\left[v_{n}(\tau)\right]^{p}}
$$


So we have that

$$
\int_{|t-\tau|>n} b(t-\tau) \frac{\left|l_{n}(t, \tau)\right|^{p}}{[V(|t-\tau| / n)]^{p-1}} d t \leq \int_{|t-\tau|>n} b(t-\tau)\left(\frac{w_{n}(t)}{w_{n}(\tau)}+1\right) d t .
$$

But now we may extend the domain of integration to all of $\mathbb{R}$, and then Lemma 3.4 (iv) shows that this is bounded (by $r^{-1}+\|b\|_{L^{1}(\mathbb{R})}$ ). So $B_{n}$ remains bounded as $n \rightarrow \infty$.

Proof of Proposition 3.2. As in Eggermont [7], we note that without loss of generality we may assume that $\mu=1$ in (3.1), and that $\lambda \neq 0$. We may then as well assume that $\lambda=1$.

Let us suppose by way of contradiction that (3.11) does not hold. Then there exist sequences $\left\{\mathscr{K}_{n}\right\} \subset \mathscr{E}$ and $\left\{x_{n}\right\} \subset L^{\infty}(\Omega)$ such that

$$
\left\|x_{n}-\mathscr{K}_{n} x_{n}\right\|_{L^{\infty}(\Omega)}=O\left(n^{-2}\right) \quad \text { as } n \rightarrow \infty \text {, }
$$

and $\left\|x_{n}\right\|_{L^{\infty}(\Omega)}=1$ for all $n$.

Observe that from (3.2) it follows that

$$
\left|\mathscr{K}_{n} x_{n}(t+h)-\mathscr{K}_{n} x_{n}(t)\right| \leq e(h)
$$

for all $n$, and all $t \in \Omega$ with $t+h \in \Omega$, so that $\left\{\mathscr{K}_{n} x_{n}\right\}_{n}$ is equi-uniformly continuous on $\Omega$. Note that from (3.14) we have that

$$
\left\|\mathscr{K}_{n} x_{n}\right\|_{L^{\infty}(\Omega)} \geq 1-O\left(n^{-2}\right) .
$$

It follows that $y_{n}=\mathscr{K}_{n} x_{n} /\left\|\mathscr{K}_{n} x_{n}\right\|_{L^{\infty}(\Omega)}$ satisfies $\left\|y_{n}-\mathscr{K}_{n} y_{n}\right\|_{L^{\infty}(\Omega)}=O\left(n^{-2}\right)$ and $\left\{y_{n}\right\}_{n}$ is equi-uniformly continuous. Since $\left\|y_{n}\right\|_{L^{\infty}(\Omega)}=1$ there exists a $t_{n} \in \Omega$ such that $\left|y_{n}\left(t_{n}\right)\right| \geq \frac{1}{2}$, and by the equi-uniform continuity there exists a $d>0$ such that

$$
\left|y_{n}(t)\right| \geq \frac{1}{3} \quad \text { for all } t \in \Omega, \quad\left|t-t_{n}\right|<d .
$$

Now consider $v_{n} \in L^{p}(\Omega)$ (by restriction from $\mathbb{R}$ to $\Omega$ ), defined in Lemma 3.4. Then

$$
\left\|v_{n} y_{n}-v_{n} \mathscr{K}_{n} y_{n}\right\|_{L^{p}(\Omega)}=O\left(n^{-2+1 / p}\right)=O\left(n^{-1}\right)
$$

as $n \rightarrow \infty$. Also,

$$
\left\|v_{n} y_{n}\right\|_{L^{p}(\Omega)}^{p} \geq \int_{\substack{\left|t-t_{n}\right| \leq d \\ t \in \Omega}}\left|\frac{1}{3} v_{n}(t)\right|^{p} d t \geq d^{*}
$$

for some $d^{*}>0$, for all $n$. Consequently,

$$
\frac{\left\|v_{n} y_{n}-v_{n} \mathscr{K}_{n} y_{n}\right\|_{L^{p}(\Omega)}}{\left\|v_{n} y_{n}\right\|_{L^{p}(\Omega)}} \rightarrow 0 \quad(n \rightarrow \infty) .
$$

Now suppose that for all $y \in L^{\infty}(\Omega)$,

$$
\left\|v_{n} \mathscr{K}_{n} y-\mathscr{K}_{n} v_{n} y\right\|_{L^{p}(\Omega)}^{p} \leq \varepsilon_{n}^{*}\left\|v_{n} y\right\|_{L^{p}(\Omega)}^{p},
$$


with $\varepsilon_{n}^{*} \rightarrow 0$; then (3.15) implies that

$$
\frac{\left\|v_{n} y_{n}-\mathscr{K}_{n} v_{n} y_{n}\right\|_{L^{p}(\Omega)}}{\left\|v_{n} y_{n}\right\|_{L^{p}(\Omega)}} \rightarrow 0 \quad(n \rightarrow \infty) .
$$

It follows that (3.10) does not hold, and the theorem is proved. So we must show (3.16). The expression on the left of (3.16) may be written as

$$
e_{n}=\int_{\Omega}\left|\int_{\Omega} k_{n}(t, \tau) l_{n}(t, \tau) z_{n}(\tau) d \tau\right|^{p} d t,
$$

where $z_{n}=v_{n} y$ and with $l_{n}(t, \tau)$ as in Lemma 3.5. Applying Hölder's inequality, we obtain $\left(\frac{1}{p}+\frac{1}{q}=1\right)$

$$
e_{n} \leq \tilde{\varepsilon}_{n} M_{n}
$$

where

$$
\tilde{\varepsilon}_{n}=\sup _{t \in \Omega}\left\{\int_{\Omega}\left|k_{n}(t, \tau)\right| V(|t-\tau| / n) d \tau\right\}^{p / q}
$$

and

$$
M_{n}=\int_{\Omega} \int_{\Omega}\left|k_{n}(t, \tau)\right|\left|l_{n}(t, \tau)\right|^{p} /[V(|t-\tau| / n)]^{p / q}\left|z_{n}(\tau)\right|^{p} d \tau d t,
$$

in which $V(t)=\min \left\{|t|^{1 /(p-1)}, 1\right\}$. Now with (3.1),

$$
\tilde{\varepsilon}_{n} \leq \sup _{t \in \Omega}\left\{\int_{\Omega} b(t-\tau) V(|t-\tau| / n) d \tau\right\}^{p / q} \leq \varepsilon_{n}^{p / q}
$$

with $\varepsilon_{n}$ as in Lemma 3.5. The last inequality comes about when $\Omega$ is replaced by $\mathbb{R}$. Thus, $\tilde{\varepsilon}_{n} \rightarrow 0$ as $n \rightarrow \infty$.

Interchanging the order of integration, we estimate $M_{n}$ as

$$
M_{n} \leq \int_{\Omega}\left|z_{n}(\tau)\right|^{p} \int_{\Omega} b(t-\tau)\left|l_{n}(t, \tau)\right|^{p}[V(|t-\tau| / n)]^{-p / q} d t d \tau
$$

and so

$$
M_{n} \leq B_{n}\left\|z_{n}\right\|_{L^{p}(\Omega)}^{p},
$$

with $B_{n}$ as in Lemma 3.5. We now have from (3.17) that

$$
e_{n} \leq \tilde{\varepsilon}_{n} B_{n}\left\|z_{n}\right\|_{L^{p}(\Omega)}^{p},
$$

with $\tilde{\varepsilon}_{n} \rightarrow 0$ and $B_{n}$ bounded as $n \rightarrow \infty$. This is (3.16).

\section{ON LINEAR CONVOLUTION-LIKE OPERATORS: THE DISCRETE CASE}

In this section we state the discrete version of Theorem 3.1, or more appropriately, the discretized version.

Let $b \in L^{1}(\mathbb{R})$ and $e \in C(\mathbb{R})$, with $e(0)=0$. Let $\Phi(b, e)$ be the set of all bounded functions $k$ on $\mathbb{Z}^{2}$ for which there exists a $\mu>0$ such that

$$
|k(m, n)| \leq \mu \int_{m-n}^{m-n+1} b(\mu t) d t
$$


and

$$
\sup _{m} \sum_{n \in \mathbb{Z}}|k(m+l, n)-k(m, n)| \leq e(\mu l) .
$$

Let $\Omega=\mathbb{Z}$ or $\mathbb{Z}^{+}$(or any subset of $\mathbb{Z}$ ). The kernel $k$ generates an operator $\mathscr{K}: l^{p}(\Omega) \rightarrow l^{p}(\Omega)$ by

$$
(\mathscr{K} x)_{m}=\sum_{n \in \Omega} k(m, n) x_{n}, \quad m \in \Omega,
$$

for $x \in l^{p}(\Omega)$, which is bounded by Young's inequality, viz.

$$
\|\cdot \mathscr{K} x\|_{l^{p}(\Omega)} \leq\|b\|_{L^{1}(\mathbb{R})}\|x\|_{l^{p}(\Omega)} .
$$

The collection of operators $\mathscr{K}$ for which $(4.1-3)$ holds is also denoted by $\Phi(b, e)$.

Theorem 4.1. Let $\mathscr{E} \subset \Phi(b, e)$ and let $\lambda \in \mathbb{C}$. If $\lambda \notin \sigma^{p}(\mathscr{K})$ for all $\mathscr{K} \in \mathscr{E}$ and

$$
\sup \left\|(\lambda \mathscr{F}-\mathscr{K})^{-1}\right\|_{l^{p}(\Omega)}<\infty
$$

for some $p>1$, then $\lambda \notin \sigma^{\infty}(\mathscr{K})$ for all $\mathscr{K} \in \mathscr{E}$ and

$$
\sup \left\|(\lambda \mathscr{J}-\mathscr{K})^{-1}\right\|_{l^{\infty}(\Omega)}<\infty .
$$

Here the suprema are taken over all $\mathscr{K} \in \mathscr{E}$.

The proof is similar to that of Theorem 3.1. The only noteworthy difference is that we cannot set $\mu=1$ in (4.1-2), so in the proof of the analogue of Proposition 3.3 we have to consider the $\mu_{n}$ for each $\mathscr{K}_{n}$ as well. Beyond notational inconvenience, this poses no problems. We omit the details.

\section{The $L^{\infty}$ Circle Condition Theorem}

We will now give the $L^{\infty}$ analogue of Theorem 2.1. As an extra assumption we require

$$
r_{\lambda} \in L^{1}\left(\mathbb{R}^{+}\right)
$$

where $r_{\lambda}$ is the resolvent defined in (2.5). It can already be seen in the linear case $g(t, y)=\lambda y$ of $(1.1)$ that this condition is necessary. The question which conditions on the kernel $k$ guarantee $(5.1)$ has received considerable attention in the literature, see Londen [13] and references therein. A classical result in this context is given by a theorem of Paley and Wiener [15] which states that (5.1) holds if $k \in L^{1}\left(\mathbb{R}^{+}\right)$and $1+\lambda K(s) \neq 0$ for $\operatorname{Re} s \geq 0$. We remark that this would also follow with Theorem 3.1 , because $\left\|(\mathscr{I}+\lambda \mathscr{K})^{-1}\right\|_{L^{2}\left(\mathbb{R}^{+}\right)}<\infty$ holds by Plancherel's formula, and $\left\|(\mathscr{I}+\lambda \mathscr{K})^{-1}\right\|_{L^{\infty}\left(\mathbb{R}^{+}\right)}<\infty$ is equivalent to (5.1). 
Theorem 5.1 (Circle Condition Theorem in $L^{\infty}$ ). Suppose (5.1) and the assumptions of the $L^{2}$ Circle Condition Theorem 2.1 hold. Then the integral equation (1.1) has a unique solution $\left.x \in L^{\infty}, \mathbb{R}^{+}\right)$for every $f \in L^{\infty}\left(\mathbb{R}^{+}\right)$. Moreover, if $x$ and $\tilde{x}$ are the solutions of (1.1) corresponding to $f, \tilde{f} \in L^{\infty}\left(\mathbb{R}^{+}\right)$, then

$$
\|x-\tilde{x}\|_{L^{\infty}\left(\mathbb{R}^{+}\right)} \leq c\|f-\tilde{f}\|_{L^{\infty}\left(\mathbb{R}^{+}\right)}
$$

for some $c<\infty$.

Remark. The $L^{\infty}$ Circle Condition Theorem permits a local version in the spirit of Liapunov stability: If we assume (2.2) to hold only in a tube around a fixed solution $\tilde{x} \in L^{\infty}\left(\mathbb{R}^{+}\right)$, i.e., in

$$
\{(t, x): t \geq 0,|x-\tilde{x}(t)| \leq \varepsilon\},
$$

then the conclusion of Theorem 5.1 remains valid provided we have that $\|f-\tilde{f}\|_{L^{\infty}\left(\mathbb{R}^{+}\right)} \leq \delta$ for some sufficiently small $\delta>0$. (This can be seen by modifying $g$ outside the tube such that it satisfies (2.2) everywhere. We then apply Theorem 5.1 and note that the solution remains inside the tube, so that it is not affected by the modification of $g$.) Such a local variant does not exist in the $L^{2}$ theory.

Proof of Theorem 5.1. We will first consider linearizations of equation (1.1) and then conclude with the help of the mean value theorem.

(a) For arbitrary measurable $z: \mathbb{R}^{+} \rightarrow \mathbb{R}$ we consider the linearization of equation (1.1) around $z$,

$$
y+k *\left(\frac{\partial g}{\partial x}(z) y\right)=f,
$$

where $\frac{\partial g}{\partial x}(z)(t)=\frac{\partial g}{\partial x}(t, z(t))$. Equivalently, $y$ is the solution of the linearized equation (2.7),

$$
y+\lambda^{-1} r_{\lambda} *\left(\frac{\partial g}{\partial x}(z)-\lambda\right) y=v,
$$

with $v=f-r_{\lambda} * f$. The proof of Theorem 2.1, with $y, \frac{\partial g}{\partial x} y, 0,0$ in the roles of $x, g(x), \tilde{x}, \tilde{v}$ respectively, shows that (see (2.8))

$$
\|y\|_{L^{2}\left(\mathbb{R}^{+}\right)} \leq \frac{l+d}{d}\|v\|_{L^{2}\left(\mathbb{R}^{+}\right)} \text {for every } v \in L^{2}\left(\mathbb{R}^{+}\right) .
$$

Now Theorem 3.1, whose assumptions are satisfied because of (2.2) and (5.1), yields

$$
\|y\|_{L^{\infty}\left(\mathbb{R}^{+}\right)} \leq c^{\prime}\|v\|_{L^{\infty}\left(\mathbb{R}^{+}\right)} \text {for every } v \in L^{\infty}\left(\mathbb{R}^{+}\right),
$$

where the constant $c^{\prime}$ is independent of the function $z$ around which we linearized.

(b) Now consider equation (2.7),

$$
x+\lambda^{-1} r_{i} *(g(x)-\lambda x)=v,
$$


with $v \in L^{\infty}\left(\mathbb{R}^{+}\right)$. By Picard iteration on finite intervals $[0, T]$ it is easily verified that there exists a unique solution $x$ which is measurable and locally bounded on $\mathbb{R}^{+}$.

Let $x, \tilde{x}: \mathbb{R}^{+} \rightarrow \mathbb{R}$ be the solutions of (5.5) corresponding to $v, \tilde{v} \in L^{\infty}\left(\mathbb{R}^{+}\right)$. By the mean value theorem, we have that

$$
g(t, x(t))-g(t, \tilde{x}(t))=\frac{\partial g}{\partial x}(t, z(t))(x(t)-\tilde{x}(t))
$$

for a certain measurable function $z$, so that $x-\tilde{x}$ satisfies the linearized equation

$$
(x-\tilde{x})+\lambda^{-1} r_{\lambda} *\left(\frac{\partial g}{\partial x}(z)-\lambda\right)(x-\tilde{x})=v-\tilde{v} .
$$

Now (5.4) implies that

$$
\|x-\tilde{x}\|_{L^{\infty}\left(\mathbb{R}^{+}\right)} \leq c^{\prime}\|v-\tilde{v}\|_{L^{\infty}\left(\mathbb{R}^{+}\right)} .
$$

Since $g(t, 0)=0$ for all $t$, the solution corresponding to $\tilde{v}=0$ is $\tilde{x}=0$, so that we also have

$$
\|x\|_{L^{\infty}\left(\mathbb{R}^{+}\right)} \leq c^{\prime}\|v\|_{L^{\infty}\left(\mathbb{R}^{+}\right)} .
$$

Finally, equation (1.1) is equivalent to equation (2.7), or (5.5), for $v=f-r_{\lambda} * f$, so that we obtain the unique solvability in $L^{\infty}\left(\mathbb{R}^{+}\right)$, as well as the Lipschitz continuity (5.2), with $c=c^{\prime}\left(1+\left\|r_{\lambda}\right\|_{L^{1}\left(\mathbb{R}^{+}\right)}\right)$.

In Corollary 2.2 we obtained an $L^{2}$ estimate which holds uniformly for all $\lambda>0$. To get $L^{\infty}$ estimates, uniformly in $\lambda$, we need that the resolvent satisfies, for all $\lambda>0$,

$$
\begin{gathered}
\left|r_{\lambda}(t)\right| \leq \mu b(\mu t), \quad \text { a.e. } t>0, \\
\int_{0}^{\infty}\left|r_{\lambda}(t+h)-r_{\lambda}(t)\right| d t \leq d(\mu h) \quad \text { for } h>0,
\end{gathered}
$$

with some $\mu=\mu(\lambda)>0$ and functions $b \in L^{1}\left(\mathbb{R}^{+}\right)$and $d \in C[0, \infty)$, with $d(0)=0$, which are independent of $\lambda$.

Corollary 5.2. Under conditions (2.9-10) and (5.7-8) the $L^{\infty}$ estimate (5.2) holds with a constant $c$ which is independent of $\lambda>0$.

This follows directly with the proof of Theorem 5.1, where now Theorem 3.1 yields $c^{\prime}$ in (5.4) which is independent of $\lambda$, and $\left\|r_{\lambda}\right\|_{L^{1}\left(\mathbb{R}^{+}\right)} \leq\|b\|_{L^{1}\left(\mathbb{R}^{+}\right)}$. Let us give frequency domain criteria which imply conditions (5.7) and (5.8).

Lemma 5.3. Let $\left\{R_{\lambda}\right\}_{\lambda \in \Lambda}$ be a family of functions which are analytic and uniformly bounded in a sector $S:|\arg s|<\varphi$ with $\varphi>\frac{\pi}{2}$, and continuous at 0 . Suppose that for every $\lambda \in \Lambda$ there exists $\mu=\mu(\lambda)>0$ such that the following estimates hold for $s \in S$ :

$$
\begin{array}{r}
\left|R_{\lambda}(s)-R_{\lambda}(0)\right| \leq L|s / \mu|^{\alpha} \text { for }|s / \mu| \leq 1, \\
\left|R_{\lambda}(s)\right| \leq M|s / \mu|^{-\beta} \text { for }|s / \mu| \geq 1,
\end{array}
$$


with $\alpha>0, \beta>0, L$ and $M$ independent of $\lambda$. Then $R_{\lambda}(s)$ are the Laplace transforms of functions $r_{\lambda}(t)$ which satisfy conditions (5.7) and (5.8) uniformly in $\lambda \in \Lambda$.

For example, for $k(t)=t^{\alpha-1} / \Gamma(\alpha)$, i.e., $K(s)=s^{-\alpha}$, the resolvent has Laplace transform $R_{\lambda}(s)=\lambda /\left(\lambda+s^{\alpha}\right)$, and for $\lambda>0$ the assumptions of Lemma 5.3 are satisfied with $\mu=\lambda^{1 / \alpha}$ and $\beta=\alpha$. They are also satisfied for $\lambda>0$ when $K(s)$ is a rational function (with $K(\infty)=0$ ) which has $\operatorname{Re} K(s) \geq 0$ for $\operatorname{Re} s \geq-\sigma$, with $\sigma>0$. This can be seen with the help of the following criterion.

Lemma 5.4. Let $1 / K(s)$ be analytic in a sector $S:|\arg s|\left\langle\varphi\right.$ with $\varphi>\frac{\pi}{2}$, and continuous at 0 . Suppose that there exist $\gamma>0, \beta \in(0,2)$ and $c>0$ such that

$$
\begin{aligned}
& \frac{1}{K(s)}-\frac{1}{K(0)}=O\left(|s|^{\gamma}\right) \quad \text { for } s \rightarrow 0 \text { in } S, \\
& \frac{1}{K(s)}=c s^{\beta}+o\left(|s|^{\beta}\right) \text { for } s \rightarrow \infty \text { in } S .
\end{aligned}
$$

Then $r_{\lambda}(t)$, defined by its Laplace tranform $R_{\lambda}(s)=\lambda K(s) /(1+\lambda K(s))$, satisfies conditions (5.7) and (5.8) for all sufficiently large positive $\lambda$.

Proof of Lemma 5.3. We may assume $R_{\lambda}(0)=0$ without loss of generality. Otherwise, we replace $R_{\lambda}(s)$ by $R_{\lambda}(s)-R_{\lambda}(0) /(1+s / \mu)$ and note that $R_{\lambda}(0) /(1+s / \mu)$ is the Laplace transform of $R_{\lambda}(0) \mu e^{-\mu t}$ which satisfies $(5.7)$ and (5.8).

We then have $\left|R_{\lambda}(s)\right| \leq B(s / \mu)$ with $B(z)=c \min \left(|z|^{\alpha},|z|^{-\beta}\right)$ for $s \in S$. Inserting this into the Laplace inversion formula

$$
r_{\lambda}(t)=\frac{1}{2 \pi i} \int_{\Gamma} R_{\lambda}(s) e^{s t} d s
$$

with contour $\Gamma:|\arg s|=\psi$ with $\frac{\pi}{2}<\psi<\varphi$, we get (5.7) with

$$
b(t)=\frac{1}{2 \pi} \int_{\Gamma} B(z)\left|e^{z t}\right| \cdot|d z|,
$$

which satisfies $b(t)=O\left(1+t^{\beta-1}\right)$ for $t \rightarrow 0$, and $b(t)=O\left(t^{-\alpha-1}\right)$ for $t \rightarrow \infty$. Similarly, we get (5.8) with

$$
d(h)=\frac{1}{2 \pi} \int_{\Gamma} \frac{B(z)}{|\operatorname{Re} z|}\left|e^{z h}-1\right| \cdot|d z|,
$$

which by dominated convergence is continuous also at 0 , with $d(0)=0$.

Proof of Lemma 5.4. Condition (5.12) implies that $R_{\lambda}(s)$ is, for sufficiently large $\lambda$, uniformly bounded in some sector of opening angle greater than $\frac{\pi}{2}$, and satisfies (5.10) with $\mu=\lambda^{1 / \beta}$. To apply Lemma 5.3, it remains to show (5.9) for small $\left|s^{\beta} / \lambda\right|$. To this end, we expand

$$
R_{\lambda}(s)=1-\frac{1}{\lambda K(s)}+\frac{1}{(\lambda K(s))^{2}}-+\cdots,
$$


which by (5.12) converges for sufficiently large $\lambda$. For $|s| \geq 1$ we have by (5.12)

$$
R_{\lambda}(s)-R_{\lambda}(0)=O\left(|s|^{\beta} / \lambda\right)
$$

while for $|s| \leq 1$ we get from (5.11)

$$
R_{\lambda}(s)-R_{\lambda}(0)=O\left(|s|^{\gamma} / \lambda\right) .
$$

This gives (5.9) with $\alpha=\beta$ if $\gamma \geq \beta$. Otherwise, a straightforward extension of the proof of Lemma 5.3, which takes account of the additional contribution for $|s| \leq 1$, gives (5.7) and (5.8) with $\mu=\lambda^{1 / \beta}$ and $b(t)=O\left(t^{-\gamma-1}\right)$ as $t \rightarrow \infty$.

\section{The $l^{\infty}$ STABILITY OF THE DisCRETIZATION}

In $\S 2$ we obtained an $l^{2}$ stability result for $A$-stable discretizations (1.2), using no assumptions other than those of the $L^{2}$ Circle Condition Theorem. We do not know if $l^{\infty}$ stability estimates exist under just the assumptions of the $L^{\infty}$ Circle Condition Theorem 5.1. Here we will show $l^{\infty}$ estimates under the frequency domain conditions of Lemma 5.3. These sectorial conditions allow us to include $A(\pi-\theta)$-stable methods with $\theta>\frac{\pi}{2}$ (and thus also methods of order greater than 2) and will also be useful for obtaining uniform convergence of optimal order over the half-line in the next section. From now on we will assume the following about the discretization method (1.3):

(6.1a) $\delta(\zeta)$ is analytic and without zeros in a neighborhood of the closed unit disk $|\zeta| \leq 1$, with the exception of a zero at $\zeta=1$.

(6.1b) $\frac{1}{h} \delta\left(e^{-h}\right)=1+O\left(h^{m}\right)$ as $h \rightarrow 0$, for some $m \geq 1$.

(6.1c) There exists $\theta<\pi$ such that $|\arg \delta(\zeta)| \leq \theta$ for $|\zeta|<1$.

Condition (6.1b) says that the method is of order $m$, and $(6.1 \mathrm{c})$ expresses that it is $A(\pi-\theta)$-stable. We can now state the main result of this section.

Theorem 6.1. Let the discretization method satisfy (6.1), and let the assumptions of the Circle Condition Theorem 2.1 hold, with condition (2.3) satisfied for $|\arg s| \leq \theta$ with $\theta$ of $(6.1 \mathrm{c})$. In addition, suppose that the Laplace transform of the resolvent, $R_{\lambda}(s)=\lambda K(s) /(1+\lambda K(s))$, is analytic in a sector $S:|\arg s|<\varphi$ with $\varphi>\theta$ of $(6.1 \mathrm{c})$, is continuous at 0 , and satisfies

$$
\begin{gathered}
R_{\lambda}(s)=O\left(|s|^{-\beta}\right) \quad \text { for } s \rightarrow \infty \text { in } S, \\
R_{\lambda}(s)-R_{\lambda}(0)=O\left(|s|^{\alpha}\right) \text { for } s \rightarrow 0 \text { in } S,
\end{gathered}
$$

for some $\alpha, \beta>0$. Then the discretized equation (1.2) has a unique solution $x=\left\{x_{n}\right\} \in l^{\infty}$ for every $f=\left\{f_{n}\right\} \in l^{\infty}$, for arbitrary $h>0$. Moreover, if $x$ and $\tilde{x}$ are the solutions of (1.2) corresponding to $f, \tilde{f} \in l^{\infty}$, then

$$
\|x-\tilde{x}\|_{l^{\infty}} \leq c\|f-\tilde{f}\|_{l^{\infty}}
$$

where $c$ is independent of $h>0$.

We remark that condition (6.2) implies

$$
\left|r_{\lambda}(t)\right| \leq c \cdot \min \left(t^{\beta-1}, t^{-\alpha-1}\right) \text { for } t>0
$$

(see Lemma 5.3), and hence $r_{\lambda} \in L^{1}\left(\mathbb{R}^{+}\right)$, which is condition (5.1). 
Corollary 6.2. Let the discretization method satisfy (6.1) and assume that for some $\lambda>0, \frac{\pi}{2} \leq \kappa<\pi$, and $\rho<\sin (\pi-\kappa)$,

$$
\begin{array}{ll}
\left|\frac{\partial g}{\partial x}-\lambda\right| \leq \rho \lambda & \text { everywhere, } \\
|\arg K(s)| \leq \kappa & \text { for }|\arg s| \leq \theta \quad(\text { with } \theta \text { of }(6.1 \mathrm{c})) .
\end{array}
$$

In addition, suppose that $R_{\lambda}(s)=\lambda K(s) /(1+\lambda K(s))$ satisfies, uniformly for $\lambda>0$, the assumptions of Lemma 5.3 with $\varphi>\theta$. Then the $l^{\infty}$ estimate (6.3) holds with a constant which is independent of $h>0$ and $\lambda>0$.

The proofs of Theorem 6.1 and Corollary 6.2 follow the lines of the proof of Theorem 5.1. The only additional difficulty is the verification of the conditions of Theorem 4.1, uniformly in $h$ (and $\lambda$ ). The following lemma shows that they are implied by (6.1) and the assumptions of Lemma 5.3.

Lemma 6.3. Let $\delta(\zeta)$ satisfy (6.1). Suppose that $\left\{R_{\lambda}\right\}_{\lambda \in \Lambda}$ is a family of functions which fulfill the conditions of Lemma 5.3 with $\varphi>\theta$ of (6.1). Then the coefficients in the expansion (cf. (1.3))

$$
R_{\lambda}\left(\frac{\delta(\zeta)}{h}\right)=h \sum_{n=0}^{\infty} r_{\lambda}(n, h) \zeta^{n}
$$

satisfy for $h>0, \lambda \in \Lambda$ :

$$
h\left|r_{\lambda}(n, h)-r_{\lambda}(n h)\right| \leq \begin{cases}C h \mu b(n h \mu) \cdot n^{-m} & \text { for } h \mu \leq 1, \\ C n^{-\alpha-1} \cdot n^{-m} & \text { for } h \mu \geq 1,\end{cases}
$$

for all $n \geq 1$, where $b(t)=t^{\beta-1}$ for $t \in(0,1), b(t)=t^{-\alpha-1}$ for $t \geq 1$. The numbers $\mu=\mu(\lambda), \alpha, \beta>0$ are those of Lemma 5.3, and $m$ is the order of the method given by (6.1b). Further,

$$
h \sum_{n=0}^{\infty}\left|r_{\lambda}(n+l, h)-r_{\lambda}(n, h)\right| \leq\left\{\begin{array}{ll}
d(\operatorname{lh} \mu) & \text { for } h \mu \leq 1 \\
C & \text { for } h \mu \geq 1
\end{array},\right.
$$

for all $l \geq 1$, where $d \in C[0, \infty)$, with $d(0)=0$, is independent of $h$ and $\lambda$, as is also the constant $C$ in (6.4-5).

Remark. For $n=0$ we have the bound

$$
h\left|r_{\lambda}(0, h)\right| \leq \begin{cases}C(h \mu)^{\beta} & \text { for } h \mu \leq 1, \\ C & \text { for } h \mu \geq 1,\end{cases}
$$

which follows with $h r_{\lambda}(0, h)=R_{\lambda}(\delta(0) / h)$ and (5.10).

Proof of Lemma 6.3. (a) By Cauchy's integral formula,

$$
R_{\lambda}\left(\frac{\delta(\zeta)}{h}\right)=\frac{1}{2 \pi i} \int_{\Gamma} \frac{R_{\lambda}(s)}{(\delta(\zeta) / h-s)} d s
$$


with $\Gamma:|\arg s|=\psi$, where $\theta<\psi<\varphi$. We denote the $n$th coefficient of the power series $(\delta(\zeta)-z)^{-1}$ by $e(n, z)$ and thus have

$$
r_{\lambda}(n, h)=\frac{1}{2 \pi i} \int_{\Gamma} R_{\lambda}(s) e(n, h s) d s,
$$

which resembles the Laplace inversion formula

$$
r_{\lambda}(n h)=\frac{1}{2 \pi i} \int_{\Gamma} R_{\lambda}(s) e^{n h s} d s .
$$

Our arguments will rely on the following estimate which was derived in the proof of Theorem 4.1 in Lubich [14, see (4.5), (4.13)]:

$$
|e(0, z)| \leq \frac{C}{1+|z|} \text { for } z \in \Gamma \text {, }
$$

$$
\left|e(n, z)-e^{n z}\right| \leq C\left(\left|z^{m} e^{n z / 2}\right|+\frac{\rho^{n}}{1+|z|}\right) \text { for } z \in \Gamma, n \geq 1,
$$

with some $0<\rho<1$. We may again suppose $R_{\lambda}(0)=0$ without loss of generality. Otherwise, we replace $R_{\lambda}(s)$ by $R_{\lambda}(s)-R_{\lambda}(0) /(1+s / \mu)$ and will observe that the coefficients of

$$
R_{\lambda}(0) /\left(1+\frac{\delta(\zeta)}{h \mu}\right)=R_{\lambda}(0) h \mu \sum_{n=0}^{\infty} e(n,-h \mu) \zeta^{n}
$$

satisfy (6.4-5).

(b) We get from (6.7-8) and (6.10) with the substitution $w=n z=n h s$

$$
\begin{aligned}
& h\left|r_{\lambda}(n, h)-r_{\lambda}(n h)\right| \leq C(h \mu)^{-\alpha} n^{-\alpha-m-1} \int_{|w| \leq n h \mu}|w|^{\alpha+m}\left|e^{w / 2}\right| \cdot|d w| \\
& +C(h \mu)^{\beta} n^{\beta-m-1} \int_{|w,| \geq n h \mu}|w|^{-\beta+m}\left|e^{w / 2}\right| \cdot|d w| \\
& +C \rho^{n} \min \left(1,(h \mu)^{\beta}\right) \text {. }
\end{aligned}
$$

The first integral dominates the second one for $n h \mu \geq 1$, where it is bounded by $C h \mu(n h \mu)^{-\alpha-1} n^{-m}$, whereas the second integral is dominating for $n h \mu \leq 1$, where it is bounded by $C h \mu(n h \mu)^{\beta-1} n^{-m}$. Together with the $\rho^{n}$ term, we therefore have (6.4).

(c) The estimate for $h \mu \geq 1$ in (6.5) follows from the corresponding estimate in (6.4) and (6.6). It remains to consider the case $h \mu \leq 1$. We have

$$
\begin{aligned}
& h \sum_{n=0}^{\infty}\left|r_{\lambda}(n+l, h)-r_{\lambda}(n, h)\right| \\
& \quad \leq \frac{1}{2 \pi} \int_{\Gamma}\left|R_{\lambda}(s)\right| \cdot h \sum_{n=0}^{\infty}|e(n+l, h s)-e(n, h s)| \cdot|d s| .
\end{aligned}
$$


We note that by (6.9) and (6.10), $|e(l, z)-e(0, z)| \leq C /(1+|z|)$, and

$$
\begin{aligned}
& \sum_{n=1}^{\infty}|e(n+l, z)-e(n, z)| \\
& \quad \leq \sum_{n=1}^{\infty}\left|e(n+l, z)-e^{(n+l) z}\right|+\sum_{n=1}^{\infty}\left|e(n, z)-e^{n z}\right|+\sum_{n=1}^{\infty}\left|e^{(n+l) z}-e^{n z}\right| \\
& \quad \leq C \frac{\left|z^{m} e^{z / 2}\right|}{1-\left|e^{z / 2}\right|}+\frac{C}{1+|z|}+\left|e^{z}\right| \cdot \frac{\left|1-e^{l z}\right|}{1-\left|e^{z}\right|} .
\end{aligned}
$$

We insert this into (6.11) and find that the contribution of the first two terms is bounded by $C(h \mu)^{\beta}$. For the last term we have

$$
\int_{z \in \Gamma:|z| \leq h \mu}(h \mu)^{-\alpha}|z|^{\alpha}\left|e^{z}\right| \frac{\left|1-e^{l z}\right|}{1-\left|e^{z}\right|}|d z| \leq C \operatorname{lh} \mu,
$$

which follows with

$$
\frac{\left|1-e^{l z}\right|}{1-\left|e^{z}\right|} \leq C l
$$

and

$$
\int_{z \in \Gamma:|z| \geq h \mu}(h \mu)^{\beta}|z|^{-\beta}\left|e^{z}\right| \frac{\left|1-e^{l z}\right|}{1-\left|e^{z}\right|}|d z| \leq C(\operatorname{lh} \mu)^{\varepsilon} \quad \text { with } 0<\varepsilon<\beta,
$$

which follows with

$$
\frac{\left|1-e^{l z}\right|}{1-\left|e^{z}\right|} \leq C l^{\varepsilon}|z|^{\varepsilon-1}
$$

This gives finally (6.5).

\section{UNIFORM ERROR ESTIMATES OVER THE HALF-LINE}

We take up again the discretized equation (1.2) in which we specify the righthand side as

$$
x_{n}+h \sum_{j=0}^{n} k(n-j, h) g\left(t_{j}, x_{j}\right)=f\left(t_{n}\right)-h \sum_{j=0}^{J} \kappa(n, j, h) g\left(t_{j}, x_{j}\right) .
$$

The right-most term represents a correction of the convolution quadrature which is to take account of the behavior of $g(t, x(t))$ near $t=0$. Here, $J$ is a fixed (and usually small) integer, and the $\kappa(n, j, h)$ are correction weights whose construction will be described below.

Let us now collect the assumptions under which we will show uniform convergence of $x_{n}$ to $x\left(t_{n}\right)$ over the half-line: The discretization method (1.3) is to satisfy conditions (6.1). It is thus of order $m$ and $A(\pi-\theta)$-stable. Furthermore:

(7.2) Equation (1.1) has a solution $x \in C^{m}[0, \infty)$ with $x^{(m)} \in L^{\infty}\left(\mathbb{R}^{+}\right)$, corresponding to $f \in C^{m}\left(\mathbb{R}^{+}\right)$with $f^{(m)} \in L^{\infty}(\tau, \infty)$ for every $\tau>0$. 
(7.3a) $g$ is $C^{m}$, and $d^{m} g(t, x(t)) / d t^{m} \in L^{\infty}\left(\mathbb{R}^{+}\right)$.

(7.3b) $g$ satisfies the circle condition $|\partial g / \partial x-\lambda| \leq 1$ in a tube around the solution $x$, i.e., in $\{(\tau, \xi): \tau \geq 0,|\xi-x(\tau)| \leq \varepsilon\}$ for some $\varepsilon>0$.

(7.4a) $k$ is locally integrable and has a Laplace transform $K$ which is analytic in a sector $S:|\arg S|<\varphi$ with $\varphi>\theta$ and satisfies

(i) $K(s)=O\left(|s|^{-\beta}\right)$ as $s \rightarrow \infty$ in $S$, for some $\beta>0$;

(ii) $s K(s)$ remains bounded as $s \rightarrow 0$ in $S$;

(iii) $R_{\lambda}(s)=\lambda K(s) /[1+\lambda K(s)]$, the Laplace transform of the resolvent, satisfies

$$
R_{\lambda}(s)-R_{\lambda}(0)=O\left(|s|^{\alpha}\right) \text { as } s \rightarrow 0 \text { in } S \text { for some } \alpha>0 .
$$

(7.4b) $K$ satisfies the circle condition

$$
|-1 / K(s)-\lambda| \geq l+d \text { for }|\arg s| \leq \theta \text {, for some } d>0 \text {. }
$$

We remark that (i) implies $k(t)=O\left(t^{\beta-1}\right)$ as $t \rightarrow 0$, and (ii) implies $k(t)=O(1)$ as $t \rightarrow \infty$. This follows easily with the Laplace inversion formula. Conditions (i) and (iii) imply (6.2). In (7.4b) it would suffice if the circle condition were satisfied only for $s=\delta(\zeta) / h$ with $|\zeta| \leq 1$ and all sufficiently small $h>0$.

For example, conditions (7.4a) (i)-(iii) are satisfied for $k(t)=t^{-1 / 2}$ or $e^{-t}$ or 1. They are not quite satisfied for $k(t)=\cos t$, whose Laplace transform $K(s)=\frac{1}{2}(s+i)^{-1}+\frac{1}{2}(s-i)^{-1}$ is a linear combination of $K_{j}\left(s+i \omega_{j}\right)$, where each $K_{j}(s)$ satisfies $(7.4 \mathrm{a})$. It requires only minor modifications to see that the following result remains valid also in such a case.

We can now state the result that we have been after.

Theorem 7.1. Under conditions (6.1) and (7.2-4) we have convergence of order $m$ uniformly over the half-line, i.e.,

$$
\left|x_{n}-x\left(t_{n}\right)\right| \leq c \cdot h^{m} \text { for } 0<h \leq h_{0} \text {, uniformly for } n \geq 0 \text {, }
$$

provided that the correction weights in (7.1) are suitably constructed.

For the proof of Theorem 7.1 we need the following approximation results. Here, $T$ is a fixed number, $0<T<\infty$.

Lemma 7.2. Under conditions (7.4a)(i) and (6.1) we have

$$
h \sum_{j=0}^{n} k(n-j, h) u\left(t_{j}\right)+h \sum_{j=0}^{m-1} \kappa(n, j, h) u\left(t_{j}\right)=(k * u)\left(t_{n}\right)+O\left(h^{m}\right)
$$

uniformly for $0 \leq n h \leq T$, for every $u \in C^{m}[0, T]$, if $\kappa(n, j, h)$ are chosen such that the above quadrature formula is exact for polynomials up to degree $m-1$. Moreover, $|\kappa(n, j, h)| \leq C(n h)^{\beta-1}$.

Lemma 7.3. Under conditions (7.4a) and (6.1) we have

$$
h \sum_{j=0}^{n} k(n-j, h) u\left(t_{j}\right)+h \sum_{j=0}^{m-2} k(n-j, h) c_{j} u\left(t_{j}\right)=(k * u)\left(t_{n}\right)+O\left(h^{m}\right)
$$


uniformly for $n h \geq T$, for every $u \in C^{m}[0, \infty)$ with $u(t)=0$ for $t \geq T / 2$, if $c_{j}$ are chosen as the correction weights of the mth order Newton-Gregory formula $(=$ end-point corrections of the trapezoidal rule).

Example. The correction weights in question are as follows.

$$
\begin{array}{ll}
c_{0}=-\frac{1}{2} & \text { for } m=2, \\
c_{0}=-\frac{7}{12}, & c_{1}=\frac{1}{12} \quad \text { for } m=3, \\
c_{0}=-\frac{5}{8}, & c_{1}=\frac{1}{6}, \quad c_{2}=-\frac{1}{24} \quad \text { for } m=4 .
\end{array}
$$

Lemma 7.4. Under conditions (6.1), (6.2) we have

$$
h \sum_{j=0}^{n} r_{\lambda}(n-j, h) v\left(t_{j}\right)=\left(r_{\lambda} * v\right)\left(t_{n}\right)+O\left(h^{m}\right),
$$

uniformly for $n h \geq 0$, for every $v \in C^{m}[0, \infty)$ with $v^{(m)} \in L^{\infty}\left(\mathbb{R}^{+}\right)$and $v=0$ in a neighborhood of 0.

Proof of Lemmas 7.2-4. (a) Lemma 7.2 is essentially Corollary 3.2 of Lubich [14]. There, an $O\left(t^{\beta-1} h^{m}\right)$ estimate was obtained, but it was only required that the quadrature formula be exact for polynomials up to degree $m-2$. Including also $t^{m-1}$ gives an $O\left(t^{\beta} h^{m}\right)$ estimate and hence the lemma.

(b) The proof of Lemma 6.3, formula (6.4), applied to $K(s)$ and with $\sim=$ -1 , shows that for $n h \geq T>0$

$$
|k(n, h)-k(n h)| \leq c n^{-m}=O\left(h^{m}\right) .
$$

With this estimate and the fact that all derivatives of $k$ remain bounded as $t \rightarrow \infty$, the result follows from classical results on Newton-Gregory quadrature formulas.

(c) Lemma 7.4 follows with the proof of Theorem 3.1 of Lubich [14].

Proof of Theorem 7.1. a) Let us first show that (7.5) holds on bounded intervals $n h \leq T<\infty$ : Using Lemma 7.2 and a Lipschitz condition for $g$, we obtain that the error $e_{n}=\left|x_{n}-x\left(t_{n}\right)\right|$ satisfies

$$
e_{n} \leq h \sum_{j=0}^{n}|k(n-j, h)| \cdot L e_{j}+h \sum_{j=0}^{m-1}|\kappa(n, j, h)| \cdot L e_{j}+O\left(h^{m}\right) .
$$

Because of

$$
\begin{aligned}
|k(n, h)| & \leq c[(n+1) h]^{\beta-1}, \quad \text { for } n h \leq T \\
|\kappa(n, j, h)| & \leq c[(n+1) h]^{\beta-1},
\end{aligned}
$$

(cf. Lemma 6.3 or see Lubich [14, (5.4)], and Lemma 7.2) a discrete Gronwalltype inequality, e.g., Brunner and van der Houwen [2], gives

$$
\left|x_{n}-x\left(t_{n}\right)\right| \leq c(T) h^{m} \text { for } n h \leq T,
$$

where $c(T)$ still grows exponentially with $T$. 
(b) Our next aim is to eliminate the correction weights $\kappa(n, j, h)$ with the help of Lemmas 7.2 and 7.3. Let $T>0$ be fixed, and choose $\phi \in C^{\infty}[0, \infty)$ such that $\phi(t)=1$ in a neighborhood of 0 and $\phi(t)=0$ for $t \geq T / 2$. We introduce the notations

$$
\begin{array}{cl}
x_{n}^{0}=\phi\left(t_{n}\right) x_{n}, & x_{n}^{+}=\left(1-\phi\left(t_{n}\right)\right) x_{n}, \\
x^{0}(t)=\phi(t) x(t), & x^{+}(t)=(1-\phi(t)) x(t), \\
g^{0}(t, x)=\phi(t) g(t, x), & g^{+}(t, x)=(1-\phi(t)) g(t, x),
\end{array}
$$

and rewrite $(7.1)$ as

$$
\begin{aligned}
x_{n}^{+}+ & h \sum_{j=0}^{n} k(n-j, h) g^{+}\left(t_{j}, x_{j}\right) \\
= & f\left(t_{n}\right)-x_{n}^{0}-h \sum_{j=0}^{n} k(n-j, h) g^{0}\left(t_{j}, x_{j}\right) \\
& -h \sum_{j=0}^{m-1} \kappa(n, j, h) g^{0}\left(t_{j}, x_{j}\right),
\end{aligned}
$$

where we choose $\kappa(n, j, h)$ as in Lemmas 7.2 and 7.3. Since $k(n, h)$ are uniformly bounded for $n h \geq T / 2$ by (7.6), we commit by (7.8) only an error of magnitude $O\left(h^{m}\right)$ if we replace $x_{j}$ by $x\left(t_{j}\right)$ and $x_{n}^{0}$ by $x^{0}\left(t_{n}\right)$ on the right-hand side. Lemma 7.3 with $u(t)=g^{0}(t, x(t))$ then gives, uniformly for $n \geq 0$,

$$
x_{n}^{+}+h \sum_{j=0}^{n} k(n-j, h) g^{+}\left(t_{j}, x_{j}\right)=v\left(t_{n}\right)+O\left(h^{m}\right)
$$

with $v(t)=f(t)-x^{0}(t)-(k * u)(t)$ which satisfies the conditions of $v$ in Lemma 7.4.

(c) We know from $\S 2$ (proof of Theorem 2.3) that equation (7.9) is equivalent to

$$
\begin{aligned}
x_{n}^{+}+ & h \sum_{j=0}^{n} \lambda^{-1} r_{\lambda}(n-j, h)\left(g^{+}\left(t_{j}, x_{j}\right)-\lambda x_{j}^{+}\right) \\
& =v\left(t_{n}\right)-h \sum_{j=0}^{n} r_{i}(n-j, h) v\left(t_{j}\right)+O\left(h^{m}\right),
\end{aligned}
$$

where the $O\left(h^{m}\right)$ term is preserved uniformly for $n \geq 0$ because of $\left\|h r_{\lambda}(\cdot, h)\right\|_{l^{1}}$ $\leq$ const, which follows from Lemma 6.3. Now we use Lemma 7.4 on the righthand side to get

$$
x_{n}^{+}+h \sum_{j=0}^{n} \lambda^{-1} r_{\lambda}(n-j, h)\left(g^{+}\left(t_{j}, x_{j}\right)-\lambda x_{j}^{+}\right)=w\left(t_{n}\right)+O\left(h^{m}\right),
$$

with $w=v-r_{\lambda} * v$. 
(d) Next we take $N$ with $T / 2 \leq N h \leq T$, and for $n h \geq T$ we split the sum as

$$
\begin{aligned}
x_{n}+ & h \sum_{j=N}^{n} \lambda^{-1} r_{\lambda}(n-j, h)\left(g\left(t_{j}, x_{j}\right)-\lambda x_{j}\right) \\
& =w\left(t_{n}\right)-h \sum_{j=0}^{N-1} \lambda^{-1} r_{\lambda}(n-j, h)\left(g^{+}\left(t_{j}, x\left(t_{j}\right)\right)-\lambda x^{+}\left(t_{j}\right)\right)+O\left(h^{m}\right),
\end{aligned}
$$

where we have noted $g^{+}=g$ and $x_{j}^{+}=x_{j}$ on the left-hand side, and have once more used (7.8) on the right-hand side. But now we observe that relation (7.11) remains valid if we replace the numerical solution $\left\{x_{n}\right\}_{n \geq N}$ by the exact solution values $\left\{x\left(t_{n}\right)\right\}_{n \geq N}$. This follows from Lemma 7.4 and the identity $x^{+}+\lambda^{-1} r_{\lambda} *\left(g^{+}(x)-\lambda x^{+}\right)=w$. So we can finally apply the $l^{\infty}$ stability estimate to conclude

$$
\left|x_{n}-x\left(t_{n}\right)\right| \leq C h^{m} \text { for } n \geq N
$$

which together with (7.8) completes the proof of the theorem.

Remark. For weakly singular kernels like $k(t)=t^{-1 / 2}$ the solution of $(1.1)$ is usually not smooth at $t=0$ as required in (7.2), but has an expansion in powers of $\sqrt{t}$ near 0 . If the correction weights are chosen such that the quadrature formula becomes exact for $t^{0}, t^{1 / 2}, \ldots, t^{m-3 / 2}, t^{m-1}$, then we still get convergence of order $m$ as in Theorem 7.1, cf. Lubich [14, §5] for the extensions of Lemmas 7.2 and 7.3 which are then needed.

\section{The Circle Condition Theorem in $L^{p}$ for $1 \leq p \leq \infty$}

Close scrutiny reveals that the main ingredient in the proof of the $L^{\infty}$ Circle Condition Theorem 5.1 is the inequality (5.4) which in effect says that the linearized integral operators in equation (5.3) have bounded inverses on $L^{\infty}\left(\mathbb{R}^{+}\right)$, uniformly in $z$. If the same statement holds in an $L^{p}$ setting, for all $1 \leq p \leq \infty$, then we have a proof of the following theorem.

Theorem 8.1 (Circle Condition Theorem in $L^{p}$ ). Let $1 \leq p \leq \infty$. Suppose that (5.1) holds in addition to the assumptions of the $L^{2}$ Circle Condition Theorem 2.1. Then the integral equation (1.1) has a unique solution $x \in L^{p}\left(\mathbb{R}^{+}\right)$for every $f \in L^{p}\left(\mathbb{R}^{+}\right)$. Moreover, if $x$ and $\tilde{x}$ are the solutions of $(1.1)$ corresponding to $f, \tilde{f} \in L^{p}\left(\mathbb{R}^{+}\right)$, then

$$
\|x-\tilde{x}\|_{L^{p}\left(\mathbb{R}^{+}\right)} \leq c\|f-\tilde{f}\|_{L^{p}\left(\mathbb{R}^{+}\right)}
$$

for some $c<\infty$.

Corollary 8.2. Under conditions $(2.9-10)$ and (5.7-8) the $L^{p}$ estimate (8.1) holds with a constant $c$ which is independent of $\lambda>0$. 
As pointed out, it suffices to prove the boundedness of the inverses of the linearized operators on $L^{p}\left(\mathbb{R}^{+}\right)$. We phrase the result in the style of $\S 3$.

Let $\Omega=\mathbb{R}$ or $\mathbb{R}^{+}$, and let $b \in L^{1}(\mathbb{R})$ and $e \in C(\mathbb{R})$, with $e(0)=0$. We consider the class of integral operators $\mathscr{T}$ defined by

$$
\mathscr{T} y(t)=\int_{\Omega} k(t, \tau) a(\tau) y(\tau) d \tau, \quad t \in \Omega,
$$

where

$$
\|a\|_{L^{\infty}(\Omega)} \leq 1
$$

and where not only $k \in \mathscr{F}(b, e)$, see $\S 3$, but also $\check{k} \in \mathscr{F}(b, e)$, in which $\check{k}(t, \tau)=k(\tau, t)$. We denote this class by $\mathscr{H}(b, e)$. As in $\S 3$, each $\mathscr{T}$ is a bounded linear operator on every $L^{p}(\Omega), 1 \leq p \leq \infty$.

Theorem 8.3. Let $1 \leq p \leq \infty$, let $\lambda \in \mathbb{C}$, and let $\mathscr{E} \subset \mathscr{H}(b, e)$. If $\lambda \notin \sigma^{2}(\mathscr{T})$ for all $\mathscr{T} \in \mathscr{E}$, and

$$
\sup \left\|(\lambda \mathscr{I}-\mathscr{T})^{-1}\right\|_{L^{2}(\Omega)}<\infty
$$

then $\lambda \notin \sigma^{p}(\mathscr{T})$ for all $\mathscr{T} \in \mathscr{E}$ and

$$
\sup \left\|(\lambda \mathscr{F}-\mathscr{T})^{-1}\right\|_{L^{p}(\Omega)}<\infty \text {. }
$$

The suprema in (8.4-5) are taken over all $\mathscr{T} \in \mathscr{E}$.

Proof. The essential tools in the proof are Theorem 3.1 for $p=2$, the M. Riesz convexity theorem, as well as a duality argument.

Since $\lambda$ cannot equal 0 , we may assume $\lambda=1$ in the following. From Theorem 3.1 we already know that

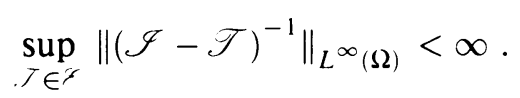

Since the operators $(\mathscr{I}-\mathscr{T})^{-1}: L^{2}(\Omega) \rightarrow L^{2}(\Omega)$ and $(\mathscr{I}-\mathscr{T})^{-1}: L^{\infty}(\Omega) \rightarrow$ $L^{\infty}(\Omega)$ coincide on $L^{2}(\Omega) \cap L^{\infty}(\Omega)$, it follows from the M. Riesz convexity theorem, Stein and Weiss [18, p. 179], that $(\mathscr{I}-\mathscr{T})$ is invertible on each $L^{p}(\Omega), 2 \leq p \leq \infty$, and

$$
\sup _{J \in \mathcal{Z}}\left\|(\mathscr{I}-\mathscr{T})^{-1}\right\|_{L^{p}(\Omega)}<\infty .
$$

We now apply the same argument to the operators $\mathscr{S}$,

$$
\mathscr{P} y(t)=\int_{\Omega} k(\tau, t) a(\tau) y(\tau) d \tau, \quad t \in \Omega .
$$

First we verify $(8.4)$ for the operators $\mathscr{F}$. Note that we may factor $\mathscr{S}$ as $\mathscr{S}=\mathscr{R} \mathscr{A}$, where $\mathscr{A} y(t)=a(t) y(t)$ and $\mathscr{R}$ is the integral operator with kernel $k(\tau, t)$. Now the operators $\mathscr{A} \mathscr{R}$ are the transposes of the operators $\mathscr{T}$, and thus have the same $L^{2}(\Omega)$ spectra and norms:

$$
\left\|(\mathscr{I}-\mathscr{A} \mathscr{R})^{-1}\right\|_{L^{2}(\Omega)}=\left\|(\mathscr{I}-\mathscr{T})^{-1}\right\|_{L^{2}(\Omega)} .
$$


Moreover, since

$$
(\mathscr{I}-\mathscr{R} \mathscr{A})^{-1}=\mathscr{I}+\mathscr{R}(\mathscr{I}-\mathscr{A} \mathscr{R})^{-1} \mathscr{A},
$$

we also obtain that

$$
\sup _{\mathscr{F}}\left\|(\mathscr{I}-\mathscr{S})^{-1}\right\|_{L^{2}(\Omega)} \leq 1+c \sup _{\mathscr{T}}\left\|(\mathscr{I}-\mathscr{T})^{-1}\right\|_{L^{2}(\Omega)}<\infty,
$$

with $c=\|b\|_{L^{1}(\Omega)}$ by Young's inequality.

We now apply Theorem 3.1 to the operators $\mathscr{S}$ to obtain that each $(\mathscr{I}-\mathscr{S})$ is invertible on $L^{\infty}(\Omega)$ and

$$
\sup _{\mathscr{F}}\left\|(\mathscr{I}-\mathscr{S})^{-1}\right\|_{L^{\infty}(\Omega)}<\infty
$$

By the M. Riesz convexity theorem, $(\mathscr{I}-\mathscr{S})$ is then invertible also on each $L^{p}(\Omega), 2 \leq p \leq \infty$, and

$$
\sup _{\mathscr{S}}\left\|(\mathscr{I}-\mathscr{S})^{-1}\right\|_{L^{p}(\Omega)}<\infty .
$$

Now the standard duality argument shows that the transposes $\mathscr{I}-\mathscr{S}^{T}$, with

$$
\mathscr{S}^{T} y(t)=a(t) \int_{\Omega} k(t, \tau) y(\tau) d \tau, \quad t \in \Omega
$$

are invertible on $L^{q}(\Omega), 1 \leq q \leq 2$, and since

$$
\left\|\left(\mathscr{I}-\mathscr{S}^{T}\right)^{-1}\right\|_{L^{q}(\Omega)}=\left\|(\mathscr{I}-\mathscr{S})^{-1}\right\|_{L^{p}(\Omega)} \quad \text { if } \frac{1}{p}+\frac{1}{q}=1,
$$

we thus have that

$$
\sup _{\mathscr{f}}\left\|\left(\mathscr{I}-\mathscr{S}^{T}\right)^{-1}\right\|_{L^{q}(\Omega)}<\infty \text {. }
$$

Finally, a reasoning similar to (8.7-8) shows that since $\mathscr{S}^{T}=\mathscr{A} \mathscr{R}^{T}$, we also have that $\mathscr{I}-\mathscr{R}^{T} \mathscr{A}=\mathscr{I}-\mathscr{T}$ is invertible, and

$$
\sup _{\mathscr{T} \in \mathscr{E}}\left\|(\mathscr{I}-\mathscr{T})^{-1}\right\|_{L^{q}(\Omega)}<\infty
$$

for $1 \leq q \leq 2$. Combined with (8.6), this is the theorem.

To prove Theorem 8.1, we may now copy the proof of Theorem 5.1, replacing every occurence of $L^{\infty}\left(\mathbb{R}^{+}\right)$by $L^{p}\left(\mathbb{R}^{+}\right)$as we go along. In particular, inequality (5.4) with $L^{p}$ norms follows from Theorem 8.3.

\section{BIBLIOGRAPHY}

1. B. A. Barnes, The spectrum of integral operators on Lebesgue spaces, J. Operator Theory 18 (1987), 115-132.

2. H. Brunner and P. J. van der Houwen, The numerical solution of Volterra equations, NorthHolland, Amsterdam, 1986.

3. J. R. Cannon, The one-dimensional heat equation, Addison-Wesley, Reading, MA, 1984.

4. C. Corduneanu, Integral equations and stability of feedback systems, Academic Press, New York, 1973. 
5. G. Dahlquist, A special stability problem for linear multistep methods, BIT 3 (1963), 27-43.

6. C. A. Desoer and M. Vidyasagar, Feedback systems: Input-output properties, Academic Press, New York, 1975.

7. P. P. B. Eggermont, Uniform error estimates of Galerkin methods for monotone Abel-Volterra integral equations on the half-line, Math. Comp. 53 (1989), 157-189.

8. R. Ghez, A primer of diffusion problems, Wiley, New York, 1988.

9. E. Hairer, Ch. Lubich and M. Schlichte, Fast numerical solution of weakly singular Volterra integral equations, J. Comput. Appl. Math. 23 (1988), 87-98.

10. I. I. Kolodner, Equations of Hammerstein in Hilbert space, J. Math. Mech. 13 (1964), 701750.

11. P. Linz, Analytical and numerical methods for Volterra equations, SIAM, Philadelphia, PA, 1985.

12. A. S. Lodge, M. Renardy and J. A. Nohel, eds., Viscoelasticity and rheology, Academic Press, Orlando, 1985.

13. S. O. Londen, On some nonintegrable Volterra kernels with integrable resolvents including some applications to Riesz potentials, J. Integral Equations 10 (1985), 241-289.

14. Ch. Lubich, Convolution quadrature and discretized operational calculus. I and II, Numer. Math. 52 (1988), 129-145 and 413-425.

15. R. E. A. C. Paley and N. Wiener, Fourier transforms in the complex domain, Amer. Math. Soc. Colloq. Publ., vol. 19, Amer. Math. Soc., Providence, RI, 1934.

16. A. C. Pipkin, Lectures on viscoelasticity theory, Springer-Verlag, New York, 1972.

17. I. W. Sandberg, A frequency domain condition for stability of feedback systems containing a single time-varying nonlinear element, Bell Sys. Tech. J. 43 (1964), 1581-1608.

18. E. M. Stein and G. Weiss, Introduction to Fourier analysis on Euclidean spaces. Princeton Univ. Press, Princeton, NJ, 1971.

19. G. Zames, On the input-output stability of nonlinear time-varying feedback systems. I and II, IEEE Trans. Automat. Control AC-11 (1966), 228-238 and 465-477.

Department of Mathematical Sciences, University of Delaware, Newark, Delaware 19716

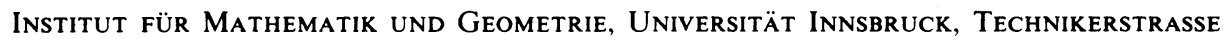
13, A-6020 Innsbruck, Austria 